\title{
Missed opportunities for improving practice performance in adult immunizations: a meta-narrative review of the literature
}

\author{
Natalia Loskutova ${ }^{1 *}$, Craig Smail ${ }^{1}$, Brian Webster ${ }^{2}$, Kemi Ajayi $^{1}$, Julie Wood ${ }^{1}$ and Jennifer Carroll ${ }^{1,3}$
}

\begin{abstract}
Background: We sought to characterize how the term "missed opportunities" is reported in the literature in the context of immunization rates and to assess how missed opportunities can be operationalized.

Methods: Peer-reviewed literature searches were conducted in April - May, 2015, to answer: "What methods research studies used to operationalize missed opportunities to vaccinate?" A meta-narrative review methodology was used.

Results: Seven studies met inclusion criteria. The methodologies for quantifying missed opportunities fell into two general categories based on: 1. the number of healthcare encounters per patient without appropriate vaccination services, defined as a number of visits per patient with no vaccination related services (Missed opportunities per patient); 2. vaccination status as "non-vaccinated" among a group of patients who had a healthcare encounter where the vaccination should/could have had happened (Missed opportunities per population).

Conclusions: Our study provided an initial overview of the methods reported in the literature, and concluded that the quantifiable missed opportunity holds promise as a measurable outcome (variable) for research and quality improvement projects aimed to increase adult immunization recommendation and uptake in primary care.
\end{abstract}

Keywords: Missed opportunity, Meta-narrative literature review, Adult immunizations

\section{Background}

Developing and providing immunizations are two of the greatest public health achievements. Adult immunization rates in US, however, remain well below Healthy People 2020 suggested targets for influenza vaccinations in adults $>65$ (target 90\%), and for pneumococcal disease in adults $\geq 65$ years (target $90 \%$ ), and in persons at high risk for pneumococcal disease aged 18-64 (target 60\%) [1]. Despite scientific advances in vaccine development, morbidity and mortality from preventable infectious diseases persist due to multiple documented barriers. Each year US primary care physicians provide more than 560 million office visits and are in the unique position to administer immunizations to patients of all ages [2]. However, health care encounters in which a person eligible to receive vaccinations is not vaccinated [3] occur in any setting where vaccinations are

\footnotetext{
*Correspondence: nloskutova@hotmail.com

${ }^{1}$ American Academy of Family Physicians National Research Network, 11400

Tomahawk Creek Pkwy, Leawood, KS 66211, USA

Full list of author information is available at the end of the article
}

offered, and these occasions are viewed as missed opportunities to provide adequate vaccination for older adults [4].

It is common in published literature on vaccination to state that missed opportunities to vaccinate exist and likely contribute to the persistently high levels of vaccinepreventable illness and gaps in achieving the populationwide Healthy People 2020 goals. In published literature, however, missed opportunities to immunize are often referred to as an abstract concept that represents the endresult of a patient not being vaccinated due to existing barriers ranging from patient resistance, provider bias, or vaccine unavailability. The term missed opportunities generally is not characterized well and is often used interchangeably with other terms that refer to poor immunization status in individual patients or populations.

Scientific evidence suggests that in order to assess and potentially change a measure, that measure or term needs to be measurable and operationalized. In the social sciences, operationalization is commonly referred 
to as the process through which abstract concepts are translated into measurable variables [5]. This suggests that missed opportunities could be potentially developed into a measure of immunizations. However, it is unknown if missed opportunities can be systematically measured in a meaningful and repeatable way. In order to address missed opportunities, there is a need to define, quantify, and track missed opportunities. From a population health perspective, operationalizing missed opportunities for immunizations could allow us to identify strategies to reduce the proportion of primary care visits that are viewed as missed opportunities to vaccinate and subsequently to close the gap on vaccinepreventable illness.

The overall goal of this work was to characterize how the term missed opportunities is reported in the literature in the context of immunization rates and to assess whether and how missed opportunities can be operationalized. Specifically, the objectives of this paper are to: 1 . characterize the missed opportunities in adult vaccinations in published articles relevant to primary care; 2 . synthesize evidence pertaining to operationalization of missed opportunities concept; and 3. explore the potential of operationalized or quantifiable missed opportunities as a measurable outcome (variable) for research and quality improvement projects focused on adult immunizations in primary care.

\section{Methods}

\section{Study overview}

This is a meta-narrative review study. We selected this method because we sought to assess and synthesize published evidence around operational definitions of missed opportunities for adult vaccinations for which, to the best of our knowledge, no operational definitions currently exist. The meta-narrative review methodology was designed specifically to summarize a research topic with no standard conceptualization (i.e., researchers bring different presumptions and goals to the research) and different paradigms (i.e., potential to explore more complex and diverse questions in one review, the possibility to include studies of different designs, utilizing multiple outcomes, varying systems, and different methodologies). Additionally, the meta-narrative review typically includes two key components: exploration of literature and the synthesis of data, resulting in the meta-narrative maps of the findings that highlight differences and similarities of various paradigms. In contrast, a systematic literature review is designed to summarize and ultimately grade the quality of evidence and the results of available carefully designed clinical trials to evaluate the evidence on the efficacy or effectiveness of healthcare interventions. Since the focus of this review was not on grading the quality of evidence related to immunization interventions but on the methodology for operationalizing a new outcome, the meta-narrative review methodology is a better fit for the objectives of the study. Thus, the meta-narrative review method was selected primarily due to the purpose of the study, the lack of standard definition of missed opportunities in the field. and the above-mentioned characteristics of the meta-narrative review methodology. We followed the meta-narrative review framework proposed by Wong, et al. [6]. We conducted a comprehensive meta-narrative review of the biomedical literature identified in the PubMed database that was published between January, 2000, and May, 2015.

\section{Literature searches}

We included studies of any design (i.e., randomized, observational, cross sectional, descriptive, qualitative), size or setting published in English in which adult immunizations was the primary outcome. We also included non-original or summarized literature (i.e., systematic or non-systematic literature reviews, commentaries).

A professional librarian employed full-time at the AAFP Leawood, KS, office, completed the initial literature search in April - May, 2015. To start the search, the following key words were used: immunizations; missed opportunities; vaccination; immunization schedule; and physician practice patterns.

The overall eligibility criteria were as follows:

- the study cohort consisted of adults 18 and older

- the focus of the study was on adult immunizations

- the study was completed in 2000 or later

- the study was published in English

Since the term missed opportunities is not a term in the $\mathrm{MeSH}$ controlled vocabulary (and hence is not included as a PubMed keyword), we searched the term in both the title and abstract of indexed articles. We also searched with conceptually related $\mathrm{MeSH}$ key words. From the results of both approaches, we identified records for title and abstract screening and review. See Additional file 1 for the full search strategy.

\section{Meta-narrative review process}

To characterize missed opportunities for adult immunizations, we first reviewed the titles and abstracts of papers retrieved using the aforementioned search criteria and key words. The titles and abstracts of all identified articles were reviewed by two reviewers (NL and CS) and selected for full text review based on the following: the articles should focus on immunizations in adults; the methods and/or results section of the abstracts should include evidence of or indication of use of data for missed opportunities; and/or describe methodology for 
how the term was operationalized or quantified; and/or have a numerical exemplification of missed opportunities presented in results.

Next, the records were included in the further full text review if they were deemed relevant to the objectives of the study based on full or partial alignment with the inclusion criteria. For those records, full text copies were obtained and included in full review. The articles were excluded if they did not provide a specific definition for missed opportunities or relevant methodology or did not include quantifiable results for missed opportunity in the abstract.

Three individuals (NL, CS, JW) reviewed the full text articles; at least two individuals reviewed each article. Full texts of these articles were reviewed using a template to summarize the study as follows: name (title, authors, date); purpose; population and setting; service or procedure; timeframe; methodology; missed opportunity definition; significant results or observations; methodological advantages and limitations. The articles were included in the results if they had a clear definition of missed opportunities, quantified or operationalized missed opportunities according to their definition or formula, and included a corresponding quantitative measure of missed opportunities in outcomes using data. The articles that originally partially aligned with the guiding review principals that did not provide additional information to satisfy all requirements including a specific definition for missed opportunities, relevant methodology or did not include quantifiable results for missed opportunity in the full text were excluded. Additionally, we reviewed reference lists from eligible studies and conducted additional searches for publications that cited eligible studies. The full texts of these articles were reviewed following the same process as above. The literature search and data extraction were completed in September, 2015.

\section{Results}

\section{Literature search and selection results}

The literature search for the systematic review identified 80 possibly relevant citations. We included seven studies. The study selection process is described in Fig. 1.

\section{Overview}

A total of seven articles were included in the final sample. Of the total, two studies focused on influenza vaccinations only, four studies focused on pneumococcal and influenza vaccinations and one study included influenza, pneumococcal and tetanus adult vaccinations. The studies enrolled a combined total of 15,140 participants. The patient populations for the majority of the studies were individuals aged 65 years

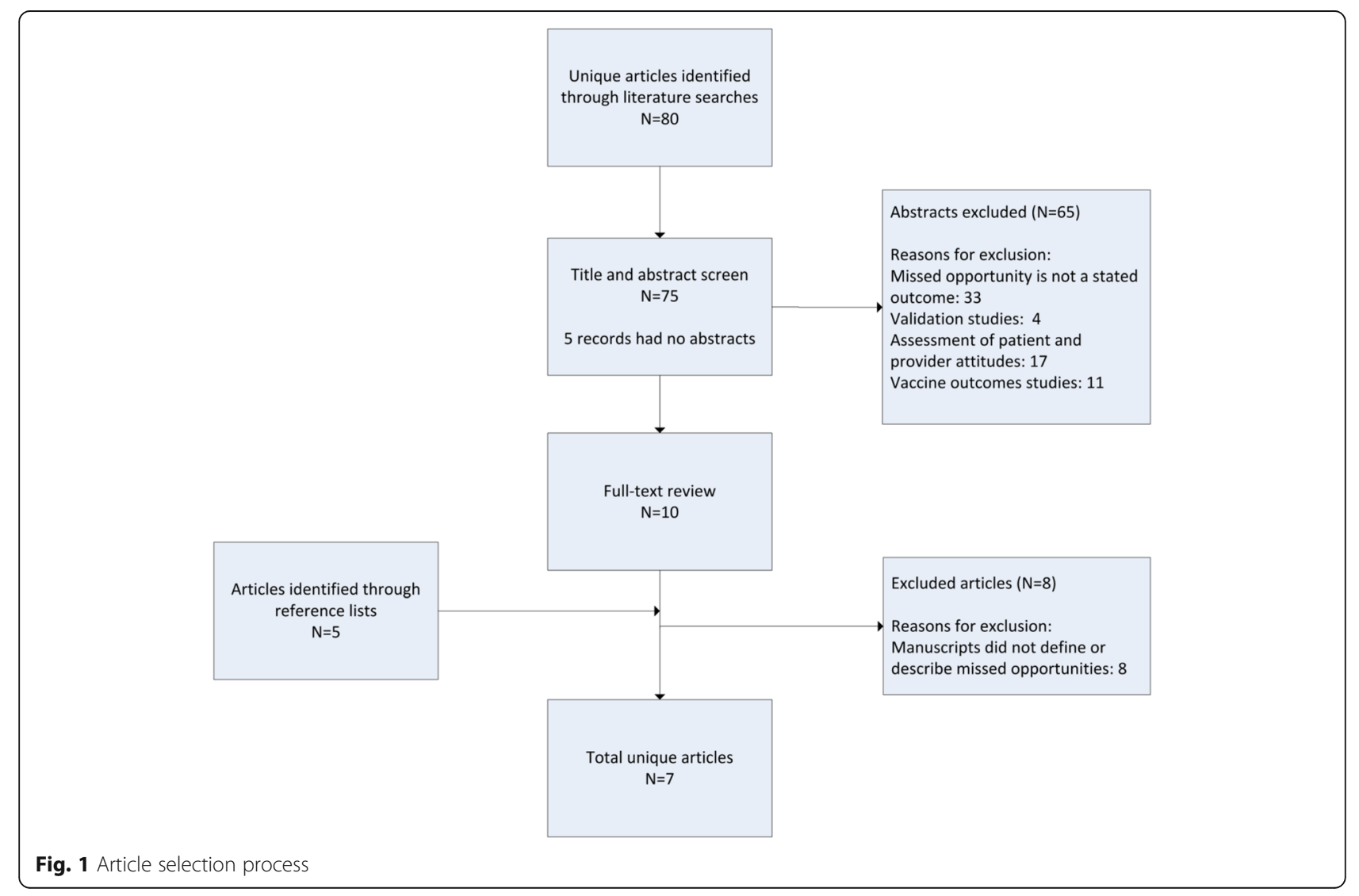


or older. The patients studied were from a variety of backgrounds including health centers in disadvantaged neighborhoods, Veterans Administration clinics, rural and urban ambulatory practices and hospitals. All studies utilized observational designs - four studies extracted data from retrospective reviews of medical records, one study had direct observations at the time of visit, and three studies incorporated surveys or interview data. Of the total, three studies were conducted in inpatient hospital settings and two were conducted in the ambulatory primary care. The summary of studies is presented in Table 1.

\section{Missed opportunities to immunize operational methodologies}

The studies provided variable detail in how the authors defined and determined missed opportunities. Generally, missed opportunity definitions were defined based on data including either visits (encounters), $[7,8]$ or a combination of patient vaccination status with encounters [9-13] (Table 1).

The most common definition of missed opportunities was estimated retrospectively; chart reviews or surveys were conducted to determine the vaccination status using reports or records of a past visit to a health care facility (Table 1). For example, Murer, et al. estimated missed opportunities based on the proportion of unvaccinated and amenable patients with at least one office visit between October and December, 2008, derived from the retrospective survey data [10].

In one study, missed opportunities were also estimated based on real-time visit observation and reported as a dichotomous outcome of either no vaccine recommendation (missed opportunity) or vaccine administration at the visit (no missed opportunity) [7].

Five of the seven studies did not take into account patients' willingness or refusal for vaccination when determining missed opportunities [7, 9, 11-13]. Nowalk, et al. incorporated patient refusal into their definition of missed opportunities by reporting visits where the vaccine was not discussed, not given, or not offered and refused by the patient. In that study, missed opportunities defined as above were quantified as the number of visits per patient [8]. Maurer, et al. incorporated patient willingness to be vaccinated in the report [10].

Variations in study design additionally included the length of observation period (from one to 5 years) and the type of healthcare encounter, ranging from a visit to a health care provider, a family physician consult, a hospital outpatient department, an emergency room to hospitalizations.
Data synthesis and meta-narrative maps of the common methodological approaches in defining missed opportunities

Here we describe a framework for operationalizing the missed opportunity term based on how the missed opportunity operational methods may be clustered given common methodological characteristics described in the included studies. We synthesized the methodologies identified in the studies and generated the meta-narrative maps of the findings with overall summary of how a "missed opportunity" term may be potentially operationalized (Fig. 2).

Upon data synthesis, the reported methodologies for quantifying missed opportunities fell into two general categories: 1. Based on the number of healthcare encounters without appropriate vaccination services, defined as a number of visits per patient with no vaccination related services (missed opportunities per patient or Patient-level) 2. Based on vaccination status as "nonvaccinated" among a group of patients who had a health care encounter where the vaccination should/could have had happened presented as proportion of nonvaccinated patients who had an encounter with health care providers (missed opportunities per patient population or Population-level).

From the outcome measure perspective, the studies that used the former patient-level method generated a specific and detailed variable that was characterized by a nominal value per patient presented for a group as a mean and SD, distinctly different form currently used measures of vaccination coverage, i.e., proportion or percentage. The population-level method resulted in a dichotomous variable at the patient level present at the group (population) level as percentage, similar in format to the commonly used vaccination coverage metrics. The population-level missed opportunity measure is, however, distinctly different from the general population metric of vaccine coverage as it assumes the documented health care encounter during a specific time frame, while the vaccination rates do not take in consideration whether the patient actually visited the health care facilities or interacted with the health care.

\section{Discussion}

The results of this study highlighted that missed opportunity is variably defined, indirectly assessed if at all, and does not appear to address or connect in a systematic way to what is known about the barriers to immunization. The results of our study, however, provide initial evidence that the missed opportunity term is operational based on the methodologies identified in several existing studies. Moreover, it can be operationalized or quantified as a measurable outcome (variable) 
Table 1 Descriptive table summary of the studies that used data derived missed opportunity for clinical or preventative service or procedure. Observational, review and interventional studies in any clinical topic area are included

\begin{tabular}{llll}
\hline $\begin{array}{l}\text { Study purpose; Population } \\
\text { and setting; Service or }\end{array}$ & $\begin{array}{l}\text { Methodology (study design, } \\
\text { pealth IT use, how the data }\end{array}$ & $\begin{array}{l}\text { Missed opportunity definition } \\
\text { (data elements used; formula }\end{array}$ & $\begin{array}{l}\text { Significant results or } \\
\text { observations } \\
\text { Location }\end{array}$ \\
were obtained) & $\begin{array}{l}\text { or other for deriving the } \\
\text { value for missed } \\
\text { opportunities) }\end{array}$
\end{tabular}

Nowalk, et al., 2004 [8]

Purpose: To identify missed opportunities and find documentation of immunizations in medical records.

810 participants age $\geq$

66 years are enrolled from 3 health centers located in disadvantaged neighborhoods. Sites include 3 VA clinics; 8 rural practices; 9 rural/urban clinics, all belonging to the same health network. Service:

Adult immunizations (influenza, pneumococcal, tetanus)

Review of visit data: 1998-2000

for influenza; 1995-2000 for PPV and tetanus

Location: USA

Singleton, et al., 2005 [11]

Purpose: To assess influenza and pneumococcal immunization rates (and racial disparities thereof) by telephone interview of adults age $65+.1839$ adult respondents (age 65+) completed a telephone questionnaire. Service: Influenza and pneumococcal vaccinations Timeframe for Influenza: 20022003 season; timeframe for pneumococcal questions: "recently, or ever, vaccinated" Location: USA
Retrospective review of paper For Tetanus and PPV: medical records. Exclusion Administered to all patients criteria for review: patient data were excluded if the date of the first visit was after the recorded date of the vaccine receipt or if the patient was not seen during the review period (i.e., 19982000 for influenza; or 19952000 for PPV/tetanus) without recorded vaccination: missed opportunities $=$ total visits minus any visits in which vaccine was discussed or refused.

For Influenza: visits during October-February where influenza vaccine was not given, discussed or refused.

Interview of respondents reporting past vaccinations via telephone doctors visit, but did not receive a flu shot or a recent recommendation for one Data used to classify M missed opportunity $\mathrm{O}$ : clinic visit dates, vaccination status, physician recommendations for vaccination, knowledge of vaccine recommendations

$67.8 \%$ had influenza vaccine in 2002-03 season; $60 \%$ had ever received pneumococcal (range 44\% - 63\%, depending on race, which was a significant predictor of vaccination status).

Missed opportunity rates (recent doctor visit and vaccine recommendation from provider but no vaccine): blacks, $26.9 \%$ versus 7.9\%; Hispanics, 19.9\% versus $12.1 \%$; and white nonHispanics, $16.2 \%$ versus $6.1 \%$.
Advantages: elucidated disparities in rates of missed opportunity amongst clinic type and setting (e.g., disadvantaged urban vs. suburban); good description of how missed opportunity were determined based on the visit and its outcomes. Limitations: retrospective medical record review period for influenza was shorter than other vaccinations - limits comparisons; study was conducted before electronic medical records were generally available

Advantages: good geographic spread of respondents (i.e., sampled from across USA); collected data on reasons for vaccination refusal; the results were concordant with those of other national surveys

Disadvantages: study relied on patient-reported data; known confusion with pneumococcal vaccination among respondents; did not directly measure actual opportunities for, or refusals of, vaccination; recommendation date is necessarily limited to what the patient remembers/were aware of (e.g., doctors may have neglected/chose not to share recommendation with patient)

Vaccination rate of $5 \%$ in those where an opportunity existed Missed opportunity: review of hospital records consultation with patients primary care providers; archived notes (deceased patients only)
Surveyed patients admitted to hospital with pneumococcal infection. If it was found that a patient was not vaccinated, investigators followed up with their primary care physician (and hospital encounters, if applicable) to determine number of missed opportunities (opportunity for vaccination defined as: in the 5 years preceding hospital admittance, patient had one or more of: family physician consult; attended hospital outpatient department; have been admitted to hospital).
Disadvantages: patients resided in UK (likely have different guidelines); small sample size $(n=101)$
30/101 (29.7\%)
Service: Pneumococcal vaccination Timeframe: 1990-1999

Location: UK 
Table 1 Descriptive table summary of the studies that used data derived missed opportunity for clinical or preventative service or procedure. Observational, review and interventional studies in any clinical topic area are included (Continued)

\section{Kyaw, et al., 2006 [9]}

Purpose: To characterize

vaccination status and

opportunities for vaccination

patients who had been

hospitalized with invasive

pneumococcal disease: Adults age

$18+$ with invasive pneumococcal

disease $(n=1878)$

Pneumococcal vaccination

Timeframe: 2001-2003

Location: USA

Skull, et al., 2007 [12]

Purpose: To examine missed opportunities for recommended

influenza and pneumococcal

vaccines among hospitalized

elderly patients

4772 hospitalized patients 65 and older with pneumonia

vaccinations and risk factor assessment

Timeframe: April 2000-March 2002

Location: Australia
Retrospective chart review

Missed opportunity definition: $\geq 1$ healthcare encounter (including hospitalization, ER visit, outpatient visit) in the 2 years prior to pneumococcal infection
Of 617 unvaccinated patients who were eligible for a vaccination, 566 had at least one opportunity for vaccination; during 1 year Missed opportunity $=92 \%$
Disadvantages: not limited to opportunities for vaccination at primary care setting (also included cardiologists, endocrinologists, other specialties)

\begin{abstract}
Self-reported survey of previous hospitalizations and number of doctor visits and provider-confirmed vaccination records

\section{Missed opportunity = provider- subject encounter: doctor visit in the year before hospitalization (or a number of visits, each is a missed opportunity) and/or a hospitalization to the same hospital in the past 5 years}

\section{Fontanesi, et al., 2004 [7]}

The study used critical path analysis to understand operational factors involved in influenza vaccinations in ambulatory care 16 ambulatory care settings, 666 encounters

influenza vaccinations, visits types, OCPE-S used to encode all encounters

Timeframe: October 2001-

January 2002

Location: USA

Maurer, et al., 2009 [10]

Purpose: To investigate the impact of reducing missed opportunities to vaccinate adults against influenza.

US adults $(n=5067)$ from a national survey by Knowledge Networks in Menlo Park, CA. Influenza vaccine

Timeframe: March 4-7, 2009

Location: USA
Prospective/observational - Visits that resulted in OCPE-S was used by visit vaccination or did not observers to document all (missed opportunity visits) activities of the visit and encode all encounters
Mean estimate of visits is 11.7 per year (range 0-20); $99.8 \%$ of unvaccinated patients had at least one missed opportunity within a year.

Influenza - 99.6\% missed opportunity with at least one visit

PPV - 99.8\% missed opportunity with at least one visit in 1 year for flu and 5 years for PPV

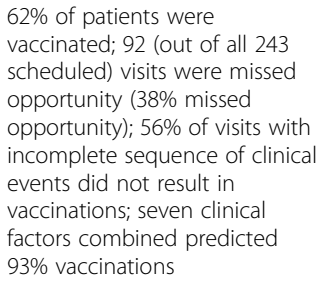
opportunity (38\% missed opportunity); $56 \%$ of visits with incomplete sequence of clinical events did not result in vaccinations; seven clinical factors combined predicted 93\% vaccinations

The study was conducted in a hospital in Australia; only patients with pneumonia were included; encounters are self-reported on a range scale with increment of 5 (0-4, 5-19, 10-14, 15-19, 20 and more) for last year and yes/no for 5 previous years; automated prompts for providers are recommended as a potential solution in discussion.

A diagram of best pathway to result in $93 \%$ vaccinations and pathways leading to missed opportunity is provided, may be useful in Q। and education strategies.

to


(1.) Patient-level

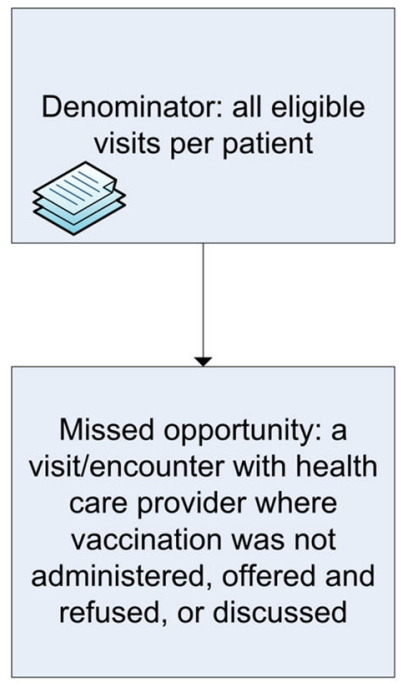

References:

Nowalk et al., 2004

Fontanesi et al., 2004

\section{(2.) Population-level}

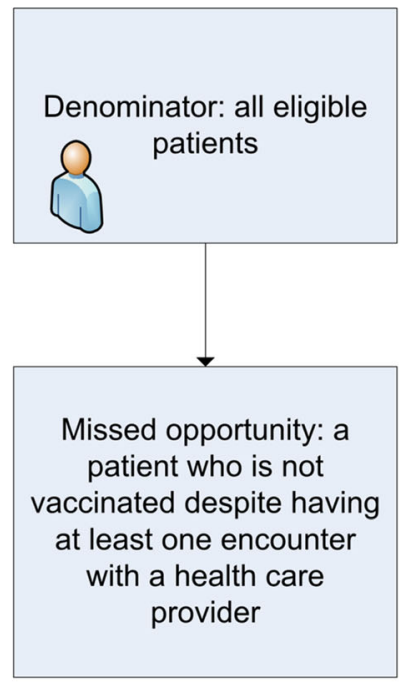

References:

Weightman et al., 2003

Kyaw et al., 2006

Skull et al., 2007

Singleton et al., 2005

Maurer, et al., 2009

\section{Footnote:}

Eligible visit: any visit with a health care provider where vaccination is feasible

Eligible patient: a patient eligible for vaccination due to age, condition, and/or vaccination history

Fig. 2 Meta-narrative maps of the common methodological approaches in operationalizing missed opportunities

that percentage in the studies we reviewed can be as high as $99.8 \%$ [12] and as such, a measure derived by this method may be prone to a ceiling effect, can only be calculated retrospectively, may not be a good measure for prospective studies, and is not very actionable because of the retrospective nature. This approach may be more suitable for simple quality improvement studies based on past performance and existing data such as Plan-Do-Study-Act (PDSA-based projects, for example. Overall, given the nascent state of research in this area, apparent lack of missed opportunity term standardization, and a limited number of studies published up to date, it is unclear how representative, accurate or comparable the approaches are based on their performance warranting a need for further research.

Despite the lack of standardization, the data synthesis revealed one common feature among all studies included in this review: that regardless of the methodology used, the missed opportunities are clearly associated with a patient encounter. That makes it easier to operationalize the term and to develop appropriate strategies, given that missed opportunities are directly tied to a patient visit with health care providers who have more control of the visits than of some other barriers to vaccinate.

While that leads to higher expectation placed on providers for stronger vaccine recommendations, [14-16] the promising aspect of addressing missed opportunities at the time when the visit actually occurs is by utilizing the care team-based strategies such as standing orders, registry-based approaches, and other team- and systemlevel efficacious intervention $[17,18]$. Focusing on one visit at a time for appropriate vaccinations for a visiting patient may be a promising quality improvement approach for increasing overall vaccinations rates among health care utilizers pending further testing.

Though investigating the potential causes of missed opportunities is outside of the scope of this study, it is important to mention that one of the reviewed studies provided initial evidence on the possible causes missed opportunities [7]. The authors of that study (Fontanesi, et al.) identified that a substantial proportion of missed opportunities in the study were explained by a combination of several factors with the most influential being an absence of provider-patient immunization discussion during a visit. 
Future studies should further explore and summarize the root causes of missed opportunities and barriers related specifically to missed opportunities as they may differ from the overall barriers to vaccinations. Further missed opportunity term operationalization will help define the currently blurry differences between "immunization barriers" and missed opportunities. We hypothesize that missed opportunities has the potential to serve as a practice performance measure due to a common characteristic identified in our study, namely, missed opportunities are clearly associated with a patient encounter. When a patient is already at the clinic, the number of applicable barriers is reduced, and yet a visit can still result in missed opportunities even when the actual opportunity, i.e., patient had a contact with healthcare provider, existed. From this perspective, missed opportunities can be viewed as a potential way to reduce barriers related to an actual encounter, such as inconsistent and weak provider recommendations, provider bias, patient resistance and clinic inefficiency.

This study also uncovered some of the areas that the future research studies need to address for successful use of operationalized missed opportunities as a measurable outcome (variable) for research and quality improvement projects focused on adult immunizations in primary care.

For example, none of the studies correlated missed opportunities with vaccination rates, were interventional in nature, or provided any normative data or recommendations on how to assess changes in missed opportunities over time. Future investigations addressing lack of standardization around operationalizing and computing missed opportunities could lead to such benefits as saving time and effort by deploying the top-ranked method(s) and design data collection and analysis plans accordingly in research and quality improvement projects. However, in order to compare outcomes of different projects reliably using missed opportunities as an additional vaccination measure and to assess performance and ranking of methods, it is essential to conduct further validation studies of this measure.

Furthermore, it is unclear if vaccination setting is an important contributing factor in the level of missed opportunities as most studies included not only visits to primary care providers but emergency room and hospitalizations, and significant missed opportunities were identified in all settings. Additional variability in how the studies included and defined what constituted a patient visit/encounter needs to be considered in the future as some studies included varying numbers of visits per time period to various settings and encounters where the vaccines were possibly offered but refused by the patient. The studies in this review used various inclusion criteria for what constituted an eligible patient encounter with health care and ranged from scheduled visits with family physicians to hospitalizations. Further standardization of the encounter characteristics will be necessary to improve research and quality improvement methods and accuracy and comparability of results across various efforts. Eligible encounter definition will need to account for several aspects including the type of visits and whether a particular visit is an appropriate opportunity to vaccinate; the type of patients and whether the visiting patient is eligible for certain vaccinations; and the target provider or health care setting and whether the visit can be linked to the provider or the health care site of interest. The development of comparable and validated timeframes for missed opportunities will be useful as the duration of patient encounter observation periods in the absence of rigid vaccination schedules recommended in adults compared to vaccination schedules in children may affect accuracy of estimations in adults.

Furthermore, future studies may need to identify and compare effective interventions and models designed to reduce missed opportunities and ultimately improve vaccination rates among adults.

\section{Strengths and limitations of present study}

Only those studies published between January, 2000, and May, 2015, were included in the literature search; therefore, recent studies in-press at time of review or published later were not included. This may in particular reduce the number of studies included in the review that compute missed opportunities using electronic medical record data and other contemporary approaches. Furthermore, we used the term "missed opportunities" as a keyword for the literature search, but it is possible that - owing to the lexical preferences of authors - other terms are in use with the same intended meaning. We performed an exhaustive, wideranging literature search of the PubMed database to identify relevant articles matching an extensive list of search terms (Additional file 1). A follow-on literature review was conducted using author citations and bibliographies of articles that passed our selection criteria. Taken together, we are confident that this approach captured all identifiable articles dealing with missed opportunities in adult immunizations published in the identified time-period.

There are several limitations of the present study that may affect generalizability and validity of results. First, the study focused on adult vaccinations only and as a result may not be generalizable to other patient populations. These may include populations distinct from those included in the study (e.g., children or newborns), or subgroups within the sample who exhibit distinct characteristics (e.g., elderly adults, immunocompromised patients). Additionally, it is possible that studies utilizing quantifiable missed opportunities as an outcome have been published in other areas relevant to different preventative or clinical services. 
Lastly, only a small number of studies remained after applying review inclusion criteria which may limit generalizability or inclusiveness of the classifications proposed. However, the data extracted from that small number of studies allowed us to draw initial conclusions that missed opportunity can be operationalized and hold promise as a measurable outcome (variable) for research and quality improvement projects aimed to increase adult immunization recommendation and uptake in primary care. Rather than limitation of the literature search process, these initial results indicate the need for future research in this area.

\section{Conclusions}

Our study provided an initial overview of the methods reported in the literature and concluded that the quantifiable missed opportunity holds promise as a measurable outcome (variable) for research and quality improvement projects aimed to increase adult immunization recommendation and uptake in primary care. The preliminary results of our study indicate that the missed opportunities term can be operationalized and point to the need for future studies to test quantifiable missed opportunities as a potentially clinically relevant, actionable, and specific clinical and research outcome.

\section{Additional file}

Additional file 1: Search Strategy. This lists the search strategy used by the librarian to locate papers for this review. (PDF $52 \mathrm{~kb}$ )

\section{Acknowledgements}

We would like to acknowledge the AAFP National Research Network for providing essential expertise, staff and support and Elizabeth Staton for assistance submitting this paper.

\section{Funding}

This work is supported in part by a research grant from Investigator-Initiated Studies Program of Merck Sharp \& Dohme Corp. Merck Sharp \& Dohme Corp. was not involved in the study design, data collection, data analysis and interpretation, writing or reporting of this work, and did not have any involvement in the decision to submit this article for publication. The opinions expressed in this paper are those of the authors and do not necessarily represent those of Merck Sharp \& Dohme Corp.

\section{Availability of data and materials}

Data sharing is not applicable to this article as no datasets were generated or analysed during the current study.

\section{Authors' contributions \\ $\mathrm{NL}$ and CS were involved in all stages of manuscript preparation including obtaining funding and study design; JW and KA were primarily involved in data analysis and interpretation of data, critically revising the paper for important intellectual content, and final editing of the paper. BW was primarily involved in interpretation of data and critically revising the paper for important intellectual content. BW and JC were primarily involved in interpretation of data and critically revising the paper for important intellectual content. All authors have approved the final paper.}

Ethics approval and consent to participate

Not applicable (not human subjects research).
Consent for publication

Not applicable (not human subjects research).

\section{Competing interests}

The authors declare that they have no competing interests.

\section{Publisher's Note}

Springer Nature remains neutral with regard to jurisdictional claims in published maps and institutional affiliations.

\section{Author details}

${ }^{1}$ American Academy of Family Physicians National Research Network, 11400 Tomahawk Creek Pkwy, Leawood, KS 66211, USA. 'Wilmington Health, 1202 Medical Center Drive, Wilmington, NC 28401, USA. ${ }^{3}$ Department of Family Medicine, University of Colorado School of Medicine, 12631 E. 17th Ave, Aurora, CO 80045, USA.

Received: 28 June 2017 Accepted: 5 December 2017

Published online: 22 December 2017

\section{References}

1. The Public Health Service Act. In: USC. vol. 42: Anonymous.https://www. healthypeople.gov/2020/topicsobjectives/topic/immunization-andinfectious-diseases/objectives. Accessed 18 Dec 17.

2. Campos-Outcalt D, Jeffcott-Pera M, Carter-Smith P, Schoof BK, Young HF. Vaccines provided by family physicians. Ann Fam Med. 2010:8(6):507-10.

3. Immunization Strategies for Healthcare Practices and Providers [http://www. cdc.gov/vaccines/pubs/pinkbook/downloads/strat.pdf]. Accessed 23 June 2017

4. Daniels NA, Nguyen T, Gildengorin G, Perez-Stable EJ. Adult immunization in university-based primary care and specialty practices. J Am Geriatr Soc. 2004:52(6):1007-12.

5. Social Research Glossary [http://www.qualityresearchinternational.com/ socialresearch/operationalisation.htm]. Accessed 23 June 2017.

6. Wong G, Greenhalgh T, Westhorp G, Buckingham J, Pawson R. RAMESES publication standards: meta-narrative reviews. BMC Med. 2013:11:20.

7. Fontanesi J, Shefer AM, Fishbein DB, Bennett NM, De Guire M, Kopald D, Holcomb K, Stryker DW, Coleman MS. Operational conditions affecting the vaccination of older adults. Am J Prev Med. 2004;26(4):265-70.

8. Nowalk MP, Zimmerman RK, Feghali J. Missed opportunities for adult immunization in diverse primary care office settings. Vaccine. 2004; 22(25-26):3457-63.

9. Kyaw MH, Greene CM, Schaffner W, Ray SM, Shapiro M, Barrett NL, Gershman K, Craig AS, Roberson A, Zell ER, et al. Adults with invasive pneumococcal disease: missed opportunities for vaccination. Am J Prev Med. 2006:31(4):286-92.

10. Maurer J, Harris KM, Lurie N. Reducing missed opportunities to vaccinate adults against influenza: what is realistic? Arch Intern Med. 2009:169(17):1633-4

11. Singleton JA, Santibanez TA, Wortley PM. Influenza and pneumococcal vaccination of adults aged $>$ or $=65$ : racial/ethnic differences. Am J Prev Med. 2005;29(5):412-20.

12. Skull SA, Andrews RM, Byrnes GB, Kelly HA, Nolan TM, Brown GV, Campbell DA: Missed opportunities to vaccinate a cohort of hospitalised elderly with pneumococcal and influenza vaccines. Vaccine 2007, 25(28):5146-5154.

13. Weightman NC, Walters A. Missed opportunities in vaccination of patients with subsequent pneumococcal bacteraemia. Br J Gen Pract. 2003;53(492):547-9.

14. Nowalk MP, Bardella IJ, Zimmerman RK, Shen S. The physician's office: can it influence adult immunization rates? Am J Manag Care. 2004;10(1):13-9.

15. Smith PJ, Kennedy AM, Wooten K, Gust DA, Pickering LK. Association between health care providers' influence on parents who have concerns about vaccine safety and vaccination coverage. Pediatrics. 2006:118(5):e1287-92.

16. Sevin AM, Romeo C, Gagne B, Brown NV, Rodis JL. Factors influencing adults' immunization practices: a pilot survey study of a diverse, urban community in central Ohio. BMC Public Health. 2016;16(1):424.

17. Willis BC, Ndiaye SM, Hopkins DP, Shefer A, Task Force on Community Preventive S. Improving influenza, pneumococcal polysaccharide, and hepatitis B vaccination coverage among adults aged $<65$ years at high risk: a report on recommendations of the task force on community preventive services. MMWR Recommendations and reports : Morbidity and mortality weekly report Recommendations and reports / Centers for Disease Control. 2005:54(RR-5):1-11.

18. Gannon M, Qaseem A, Snooks Q, Snow V. Improving adult immunization practices using a team approach in the primary care setting. Am J Public Health. 2012;102(7):e46-52. 